\title{
Innovative mini-hydro device for the recharge of electric vehicles in urban areas
}

\author{
Gabriella Balacco ${ }^{1}$ (D) Mario Binetti ${ }^{1} \cdot$ Valeria Caporaletti $^{2} \cdot$ Andrea Gioia $^{1} \cdot$ Luca Leandro $^{2} \cdot$ Vito lacobellis $^{1}$. \\ Carlo Sanvito $^{3} \cdot$ Alberto Ferruccio Piccinni $^{1}$
}

Received: 23 March 2018 / Accepted: 30 July 2018 / Published online: 4 August 2018

(c) The Author(s) 2018

\begin{abstract}
The aim of the present study is to evaluate the technical and economic feasibility of supplying recharge points for Electric Vehicles converting water pressure in excess into electric energy from a water distribution network. A real water distribution network was analyzed, by designing a power station consisting of three components: a Pump-as-Turbine, an ad hoc generator, and a control system. The last one controls the flow rate trend at the inlet of the Pump-as-Turbine to regulate the rotational speed corresponding to the optimal range of efficiency. Energy produced permits to supply a recharging point for Electric Vehicles on site with the aim to convert water pressure generally dissipated and, consequently, lost into a renewable energy and to reduce power grid congestion. The economic analysis confirms the convenience of the proposed power station, and overall, a comparable cost with pressure reduction valve devices normally adopted to control pressure pattern and leakages of a water distribution network.
\end{abstract}

Keywords Energy recovery $\cdot$ Pump-as-Turbine $\cdot$ Electric vehicles $\cdot$ Pressure head

Gabriella Balacco

gabriella.balacco@poliba.it

Mario Binetti

mario.binetti@poliba.it

Valeria Caporaletti

v.caporaletti@aqp.it

Andrea Gioia

andrea.gioia@poliba.it

Luca Leandro

1.leandro@aqp.it

Vito Iacobellis

vito.iacobellis@poliba.it

Carlo Sanvito

info@sanvitosrl.it

Alberto Ferruccio Piccinni

albertoferruccio.piccinni@poliba.it

1 Dipartimento di Ingegneria Civile, Ambientale, del

Territorio, Edile e di Chimica, Politecnico di Bari,

70125 Bari, Italy

2 Acquedotto Pugliese S.p.A., 70121 Bari, Italy

3 Sanvito S.r.l, 70043 Monopoli (Ba), Italy

$\begin{array}{ll}\text { Abbreviations } \\ \text { CFD } & \text { Computational fluid dynamics } \\ \text { DMA } & \text { District metered area } \\ \text { EV } & \text { Electric vehicle } \\ \text { PAT } & \text { Pump-as-Turbine } \\ \text { PRV } & \text { Pressure reducing valve } \\ \text { SOC } & \text { State of charge } \\ \text { SOH } & \text { State of health estimation } \\ \text { V2G } & \text { Vehicle to grid } \\ \text { WDN } & \text { Water distribution network } \\ N & \text { Rotational speed } \\ P & \text { Power } \\ h & \text { Head ratio } \\ q & \text { Flow rate ratio } \\ H & \text { Water pressure head } \\ Q & \text { Flow rate } \\ \text { BEP } & \text { Best efficiency point } \\ N_{s} & \text { Specific speed } \\ \eta & \text { Efficiency } \\ \text { ROI } & \text { Return of investment } \\ \text { NPV } & \text { Net present value } \\ \text { IRR } & \text { Internal rate of return } \\ \text { B/C } & \text { Benefit/cost ratio }\end{array}$




\section{Introduction}

The 2015 United Nations Climate Change Conference (COP21) in Paris defined the guidelines for a Community policy to reduce emissions. The migration to electric mobility is not only an environmental requirement, but also an energetic and productive need. Electric motors are more efficient and require less maintenance than the traditional ones, but they need a suitable "ecosystem" for their development; moreover, considering the recent Directive 2014/94/EU (better known as the AFID—Regulatory Framework for Alternative Fuels Infrastructure) on the deployment of alternative fuels infrastructure, the diffusion of charging systems will play an increasing role in the next future.

At national scale, the main objective of the "Piano Nazionale Infrastrutturale per la Ricarica dei Veicoli Alimentati ad Energia Elettrica" (Italian National Infrastructural Plan for the Recharge of Vehicles Powered by Electric Energy) aims to ensure, in Italy, the minimum levels of accessibility of recharging points for electric vehicles (EVs) (paragraph one article 17-septies of Italian Decree Law No. 83/2012).

The usefulness of EVs is undoubted in terms of reduced emissions; however, data show that, in Italy, the number of EVs is not significantly increasing. This rather slow growth is due to numerous causes, among which certainly a higher cost of EVs, the uncertainty of the state of charge (SOC), and state of health estimation ( $\mathrm{SOH}$ ) of the EVs' batteries [1] but above all the problems related to the power production. In fact, in Italy, the power production is exclusively from fossil source, since Italy has renounced to the power production from nuclear sources since 2011 . Nuclear power in Italy is a controversial topic. Italy started to produce nuclear energy in the early 1960s, but all plants were closed by 1990 following the Italian nuclear power referendum. On 2011, Italian voters passed a referendum to cancel plans for new reactors.

However, in the next few years, the growing number of EVs will implicate an important impact on the transport sector but also a key role on the power sector with an obvious increase of global power demand. A possible solution to mitigate the latter effect can be to associate a local power generation, such as renewable energy source, to the recharging points for EVs. The possibility of alternative systems for energy production would significantly increase the recharge point's number, creating the conditions for a greater incentive of the EVs demand and favoring the increase of their circulation especially in urban areas.

Currently, technical literature reports several examples that try to combine EVs and renewable sources [2], for which wind and solar energy are mainly studied. Despite these energy sources are more popular and widely used, they are weather depending and the renewable energy obtained vary widely over time [3].

It can certainly assume that new types of renewable energy must be identified in the future to compensate the increasing demand of EVs. Nevertheless, the intermitted electricity production of renewable energy sources must be considered and the future efforts will be addressed to new energy storage forms. Šare et al. [4] suggested how the vehicle batteries can be used as storage of electricity produced in excess, providing at the same time the procedures for giving back energy to the power grid during the lack of electricity production, thanks to a Vehicle-To-Grid (V2G) implementation. In spite of the advantages of a similar approach, this technology is not yet mature to completely replace the actual production of EVs.

Water is, nowadays, still considered a focal and important renewable energy source. Although hydropower generation does not generate emissions, the construction of hydropower plants can have environmental consequences, which greatly depend on their location but above all on how they are operated. On the contrary, the adoption of micro and mini hydropower technology (up to $100 \mathrm{~kW}$ ) in a water supply system is a really green application of this technology, due to the absence of collateral effects. Small turbines or pumps used as turbine (PATs), installed in specific points of a water supply system, convert the potential energy of water flowing in a pipe into electric power.

Currently, water distribution networks (WDNs) are frequently old and corrupted. There are two main ways to reduce heavy water losses: to rehabilitate older pipelines with expensive investments or to place pressure reducing valves (PRVs) able to reduce water pressure [5]. Numerous water management authorities are introducing PRVs into existing water supply network with the aim to reduce pipeline water leakage, especially during a period of minimum water demand. However, thinking in terms of clean and green energy, a virtuous energetic policy may move towards the water energy reuse instead to dissipate that.

Energy production in water supply systems has been realized on condition that the available hydraulic power is considerable and fairly constant [6] and the dissipation nodes, in an urban WDN, frequently present a large variability in flow rate and water head [7]. Ideal locations of a PAT system in a WDN depend on several parameters like daily flow rate pattern, water head, geometry of a WDN, and the presence of district metered areas (DMAs).

Economic analyses performed on the existing WDNs highlighted the convenience of a PAT instead of a turbine for a similar application. A PAT installation showed a payback period of about 2-3 years compared with a classical turbine and an annual income ranging from about 25,000 to about $50,000 € /$ year [8]. 
Nevertheless, the absence of consolidated approaches in this field implied that a similar technology still today can be reputed only a scientific exercise, although there are numerous studies conducted also on experimental sites [9-11]. Pumps manufactures, indeed, usually do not provide information about efficiency of a pump used like a turbine. Various researchers indicated the single-stage centrifugal pumps as the optimal and most convenient choice for a PAT, operating, however, in a range from low-to-medium head [12-15]. However, most of tests and experiments were conducted on horizontal single-stage pumps, although different types of pumps are available like multi-stage, vertical pumps, etc. Despite the numerous models and equations able to define the efficiency of a pump used in a reverse mode, this issue is still open and the efficiency of a pump when operating as turbine remains the main criticism that limits their application [16]. Moreover, experimental investigations are carried out to improve the PAT performances modifying impeller diameter and rotational speed. A simple reshaping of the blades of the impeller (with a blade rounding) can increase the PAT performances; in the same way, the greater size of the impeller increases its performances, due to the absence of a lateral passageway for the turbinated water (differently that in pump mode) and to a refinement of the inlet and outlet parts [17].

In the last few years, interesting and numerous efforts have been dedicated to unravelling the knot and to define general equations able to transpose the characteristic curve of a pump to the correspondent in reverse mode, adopting experimental set-up $[18,19]$, computational fluid dynamics applications (CFDs) [20-22], literature data [23], or proposing new devices [24]. Jain and Patel [25] presented a detailed state of the art about this aspect; several researchers, with the aim to analyze the behavior of a pump in a turbine mode, had defined several and sometimes really different relations for the prediction of PAT efficiency, based on specific speed in pump mode. Though, it is right ascribing to Chapallaz et al. [26] the worth of understanding, already 20 years ago, the usefulness of a pump in a reverse mode and dealing with all the numerous technical aspects which are still matter of concern today.

In a WDN, any energy production system must take into account a large variability of flow rate and pressure head, due to the daily demand pattern characterized by several demand peaks during the day [27]. Power plant design will contemplate the respect of both the necessary hydraulic conditions and the required backpressure, both in the case of an existing PRV replacement and not [28]. In this regard, it is necessary to individuate a good compromise between the available hydraulic energy and the desirable produced electrical energy.

In this context, the focus of this work is on coupling a PAT system for the recovery of excess pressure level in a
WDN with a dedicated system that supplies electric energy for the recharging points of EVs.

Recovering the excess mechanical energy in a WDN and converting it into electricity to supply the recharging points for the EVs would lead to a double advantage: on one hand, to use energy that, otherwise, would be lost and to the other to induce local administrations to secure agreements with water management authority, to implement incentive policies leading to the generation of a "green \& free" recharging points at zero energy cost.

The research idea is the definition of an innovative system for the supply of the EV recharging points, thanks to the energy recovered from a PAT coupled with an innovative generator and a dedicated control system. PATs allow the dissipation of the excess head in a WDN and the conversion of mechanical energy into electrical energy.

The strongly hoped diffusion by the current EU policies of the charging points for EVs well matches with the massive and wide presence of pipes of a WDN. Moreover, energy produced with PAT could be used "on site", without the need to be reintroduced into the power grid, reducing branch, and transformer congestion. In addition, the implementation of PAT into existing WDN has the advantage of smaller cost compared with other renewable energy sources [9].

\section{Materials and methods}

\section{Case study}

A real case study of an aqueduct is analyzed in this study. The WDN of the Casamassima (BA) town, located in Puglia (Southern Italy), was selected for a suitable application of a PAT, partially substituting two PRVs already installed, to recover energy.

A unique tank supplies the water drinking demand of Casamassima. The WDN is partitioned into three DMAs: DIS 1, DIS 2, and DIS 3. These districts were obtained by placing and closing isolation valves along certain pipes connecting one DMA to another. Pressure and flow meters placed upstream and downstream that these points provide the control of minimum (on the night) and daily flows. In this way, thanks to the pressure control, leakages can be extensively reduced.

Flow and pressure data, collected at time steps of $15 \mathrm{~min}$, were obtained from the remote control of the Puglia Aqueduct (called AQP in the following lines), which supplies the drinking needs and manages the whole WDN of Puglia.

Casamassima WDN (see Fig. 1) has a scheme with a unique pipe from the tank and the first two PRVs (DIS2 and DIS3) at the entrance of the town. The DMA DIS2 has in cascade a WDN that supplies Barialto Golf Club and several residential buildings; here, it was installed one more PRV 




Fig. 1 Network of Casamassima WDN, Italy, used as case study

with a consequently DMA called DIS 1 . Neglecting DIS 1 , due to limited inhabitants (about 658), the study was focused on the Casamassima town with its 19.860 inhabitants subdivided into 10.174 for DIS 2 and 9.685 for DIS 3. Flow rate values and pressure patterns measured downstream the tank (TANK) and downstream the two PRVs (DIS2 and DIS3) are, respectively, reported in Figs. 2 and 3. Observing both figures, it can be highlighted how every PRV is regulated with two different downstream pressure patterns: a former occurring during the day with the aim to guarantee the necessary pressure level to satisfy water demands and a latter occurring during the night, characterized by a greater pressure reduction to reduce nightly leaks. Moreover, each PRV has different pressure patterns due to different DMA' height and water drinking demand (Fig. 3).

The goal is to replace the existing PRVs with a PAT to recover all possible energy normally lost thanks to PRVs, converting the hydraulic head obtained as the difference between the upstream pressure head (TANK) and the required backpressure to satisfy water demands. To maximize energy production and at the same time to contain economic cost, the PAT system can be installed in a node located downstream the tank and upstream the two existing PRVs. Starting from this consideration and guaranteeing backpressure trends (minimum and maximum) actually obtained with PRVs and necessary to guarantee and to satisfy the minimum pressure level of water drinking demand, it was defined a pressure pattern showed in Fig. 4 adopting the PAT system. However, the use of a unique PAT implicates that it is necessary to leave a PRV for DIS 2 characterized with higher downstream pressure values.

Therefore, the PAT system was designed to operate with highly varying flow rates and pressure levels, respectively, for a flow rate between 39.59 and $80.63 \mathrm{l} / \mathrm{s}$ and of water pressure between 19.27 and $14.41 \mathrm{~m}$. The PAT system could generate in a day energy for $157 \mathrm{kWh}$ and the generated power ranges from 3.95 to $8.39 \mathrm{~kW}$, despite having assumed a cautionary PAT efficiency equal to 0.7 (Fig. 5).

\section{Pat working condition}

After analyzing the recent state-of-the-art regarding PAT performances, we adopted a simple but reliable methodology
Fig. 2 Hourly flow rate measured for the three selected points (Tank, DIS2, and DIS3)

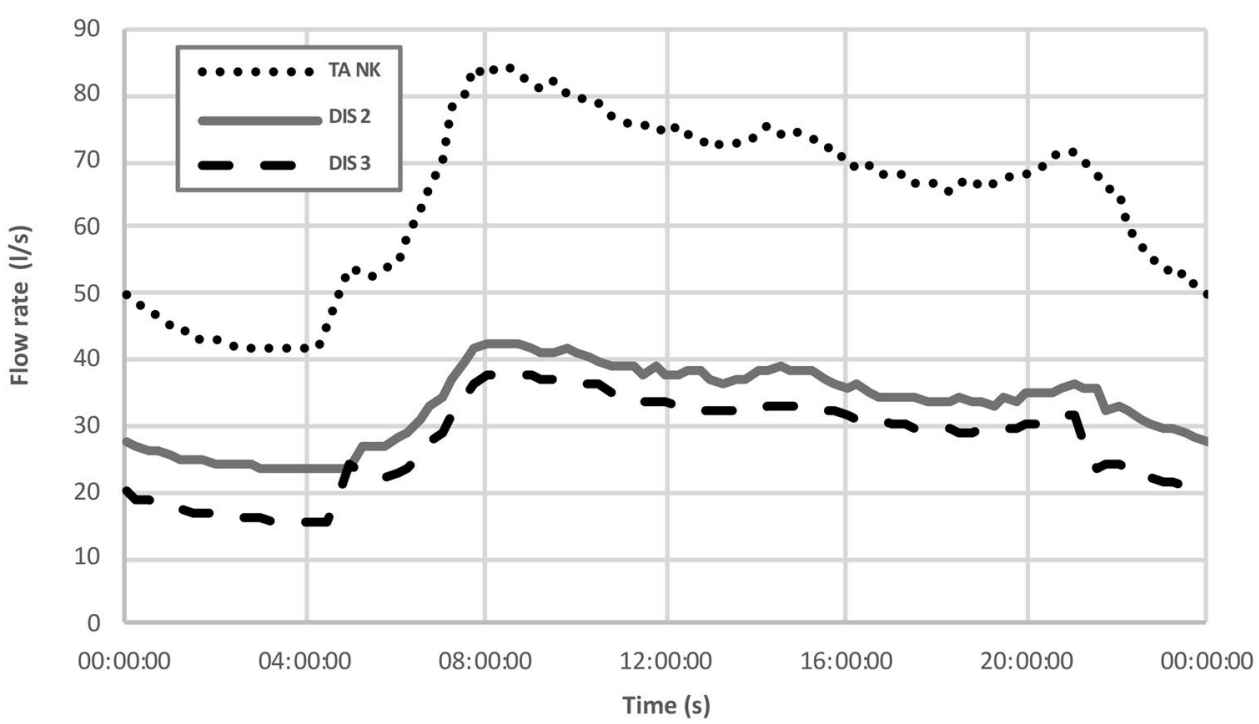



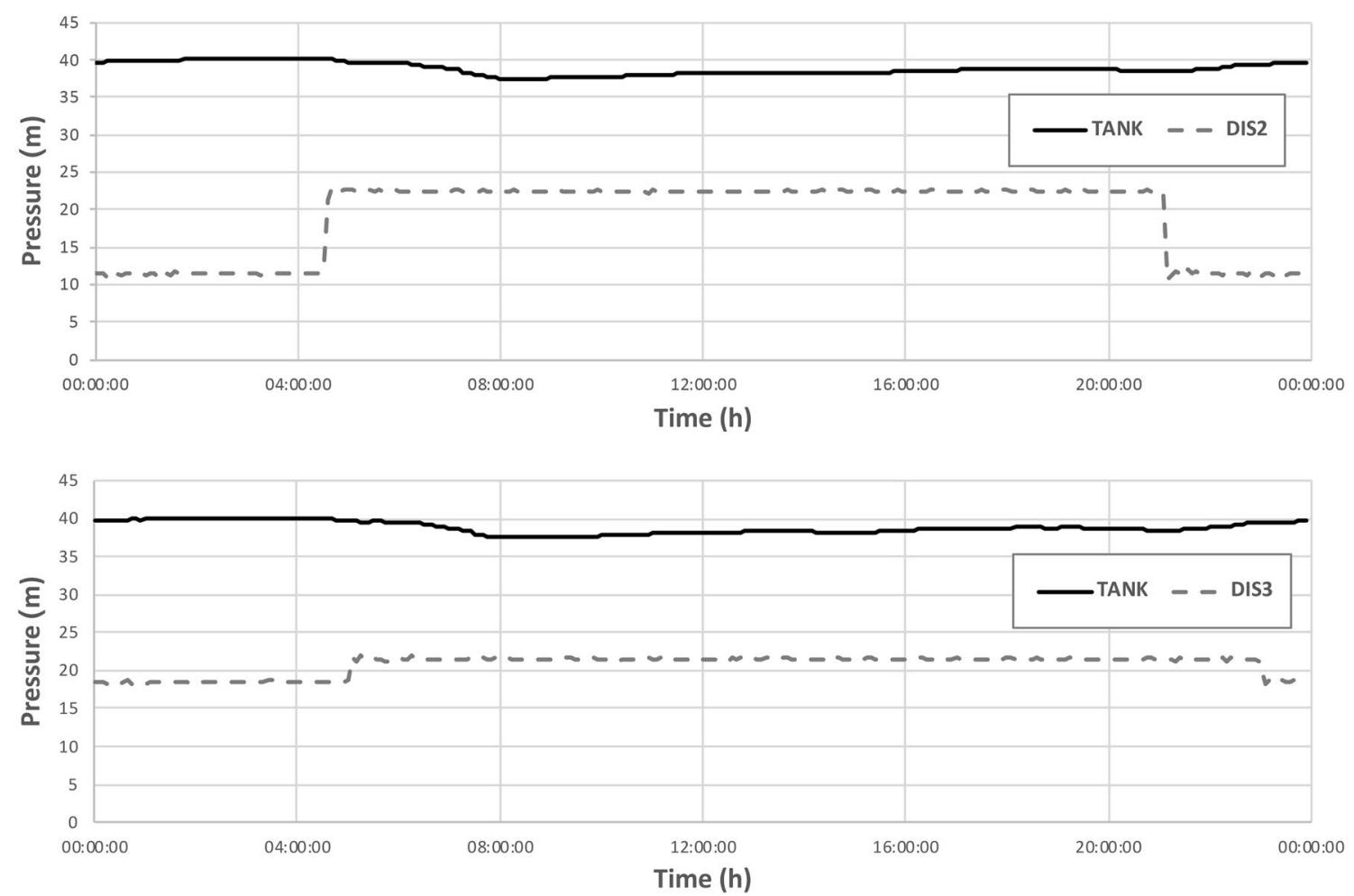

Fig. 3 Pressure patterns measured for the three selected points (TANK and, respectively, downstream the PRV valve of DIS2 and DIS3)

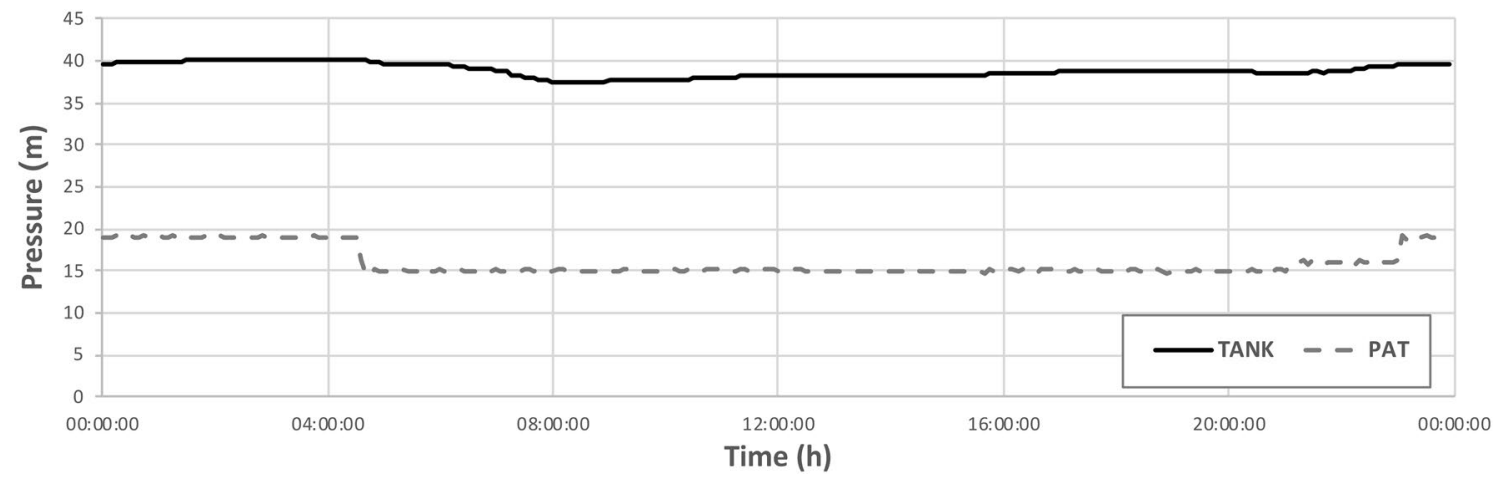

Fig. 4 Pressure pattern upstream (TANK) and downstream of the PAT system

for the PAT selection, starting from the performance of a pump in a direct way and adopting the following equations based on recently technical literature [8]. The expected errors are very limited, as showed by technical literature. The pump selected is a horizontal single-stage electric centrifugal pump (model LOWARA e-NSC 125-25/150). Pump features are summarized in Table 1, whereas its characteristic curve is represented in Fig. 5 with a grey line.

A methodology based on the definition of Best Efficiency Point (BEP) was adopted for the definition of the PAT characteristic curve. A head ratio $h$ and a flow rate ratio $q$ based on the pump characteristics were defined [e.g., 7, 29] as follows:

$h=\frac{H_{T}}{H_{p}}, q=\frac{Q_{T}}{Q_{p}}$,

where both $H$ and $Q$ are associated with BEP functioning, and subscripts are, respectively, related to pump mode $p$ and reverse mode $T$.

The specific speed of the pump equal to 39.82 was evaluated with: 


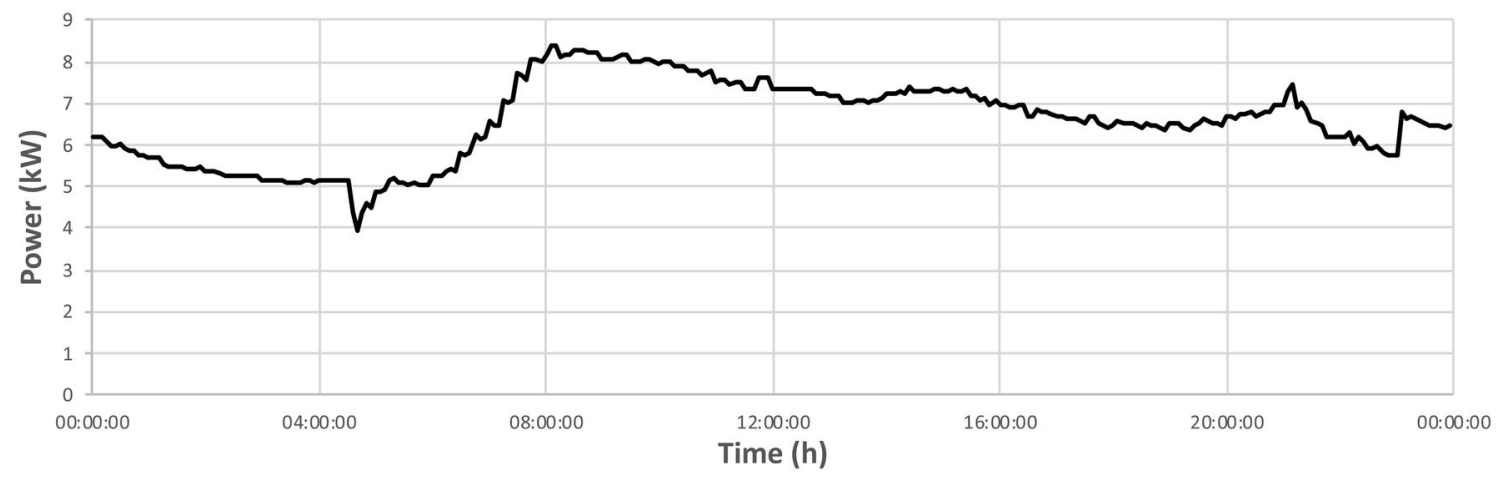

Fig. 5 Power generated by the PAT system

Table 1 Characteristics of the adopted PAT in normal mode

\begin{tabular}{ll}
\hline PAT & \\
\hline No. of poles & 4 \\
Impeller diameter D (mm) & 150 \\
Rotational speed $N(\mathrm{rps})$ & 1450 \\
Motor power $(\mathrm{KW})$ & 15 \\
Efficiency at BEP $\eta_{p}(\%)$ & 88.3 \\
Flow rate $Q_{p}$ at BEP $\left(\mathrm{m}^{3} / \mathrm{s}\right)$ & 0.07 \\
Head $H_{p}$ at BEP $(\mathrm{m})$ & 25.46 \\
\hline
\end{tabular}

$N_{\text {sp }}=N\left(\frac{Q_{P}^{0.5}}{H_{P}^{0.75}}\right)$,

where $N$ is the rotational speed of the pump at BEP.

Several researchers [29-31], testing numerous pumps, verified and reported similar trend for the Eq. (1). Starting from this analysis, Yang et al. [29] by fitting literature experimental data, related to head and discharge ratios (1) with the efficiency of the pump at BEP and proposed a relation to correlate specific speed of a pump $N_{\mathrm{sp}}$ and its corresponding value in reverse mode $N_{\mathrm{st}}$ for a single-stage centrifugal pump with a rotational speed of $1500 \mathrm{rpm}$. Equations (3) and (4) provide the estimation of $N_{\mathrm{st}}$, flow rate and head of the BEP for the PAT mode of the selected pump:

$h=\frac{1.2}{\eta_{p}^{1.1}}, q=\frac{1.2}{\eta_{p}^{0.55}}$

$N_{\text {sp }}=1.125 N_{s t}+1.73$.

Furthermore, the following Eq. (5) was adopted to define the complete characteristic curve of the PAT obtained by $[32,33]$ testing four centrifugal pumps in turbine mode; moreover the Eq. (5) was verified by Pugliese et al. [18] with a good agreement for a single-stage Lowara pump:
$\frac{H_{T}}{H_{p}}=1.0283\left(\frac{Q_{T}}{Q_{\mathrm{TB}}}\right)^{2}-0.5468\left(\frac{Q_{T}}{Q_{\mathrm{TB}}}\right)+0.5314$

Figure 6 illustrates BEP in PAT mode, characteristic curves of the selected pump for this study in direct (grey line) and reverse mode (dash-dot line), and two different characteristic curves for the PAT functioning, obtained thanks to the electric regulation better explained in the following paragraph.

\section{Installation scheme}

The power station consists of three components: a PAT, an ad hoc generator, and a control system; the PAT installation consists of a horizontal single-stage electric centrifugal pump (model LOWARA e-NSC 125-25/150), the ad hoc "Black-box Generator" is composed by electric frequency converters and a PLC-based control system is useful to control the flow rate regulation valve and the "Black-box Generator" itself. It allows the regulation of PAT' rotational speed $N$ and the measurement of generated power $P$.

The control system provides the regulation of flow at the inlet of the PAT, to set the rotational speed to an acceptable range of efficiency. The "generator", managed by the control system, gives the energy produced by the PAT to the electrical grid with values of voltage and frequency according to the grid standards itself. This peculiar "generator" system, provided by Elettronica Santerno, allows to give energy to the electrical grid with any rotational speed of the PAT.

A sketch of the PAT installation scheme is shown in Fig. 7. A by-pass of the current system was placed downstream the tank and upstream the two existing PRVs, two motorizes sectioning valves allow the introduction of the PAT system or not. Moreover, two flow rate gauges measure flow rate upstream and downstream of the PAT, with the possibility to divide flow rate between the main and the 
Fig. 6 PAT characteristic curve defined starting from the BEP
PAT characteristic curve

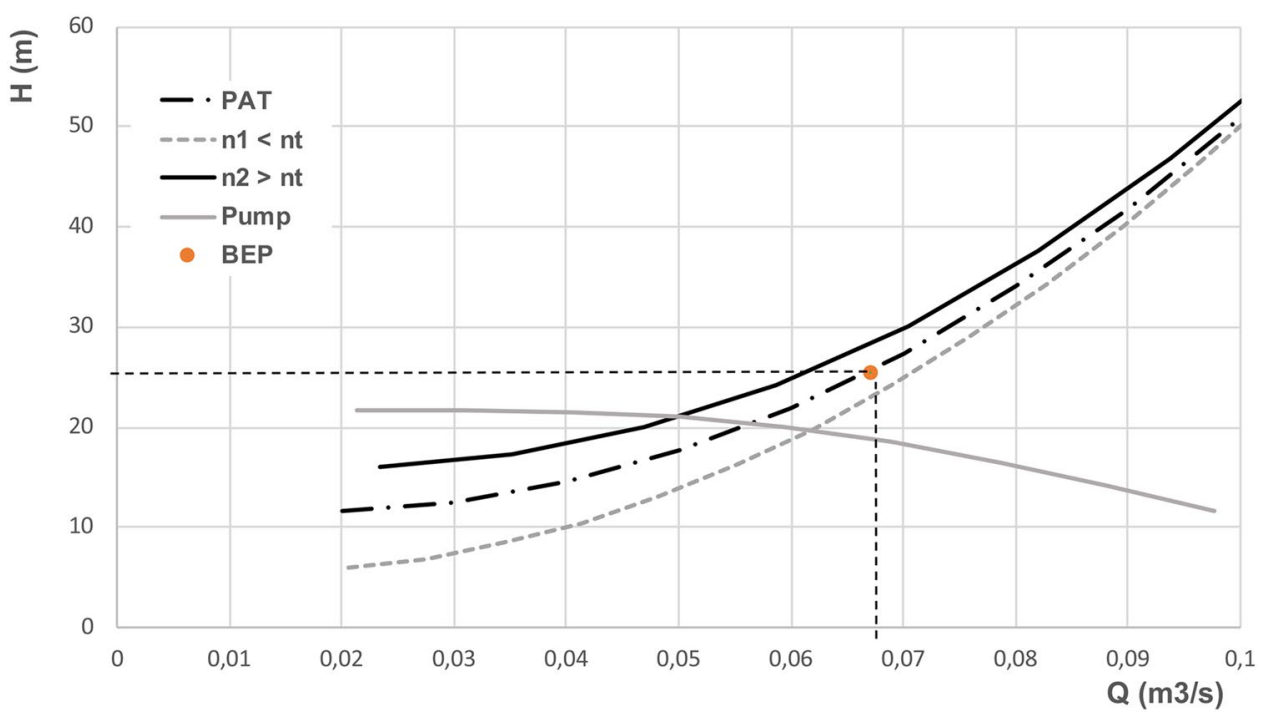

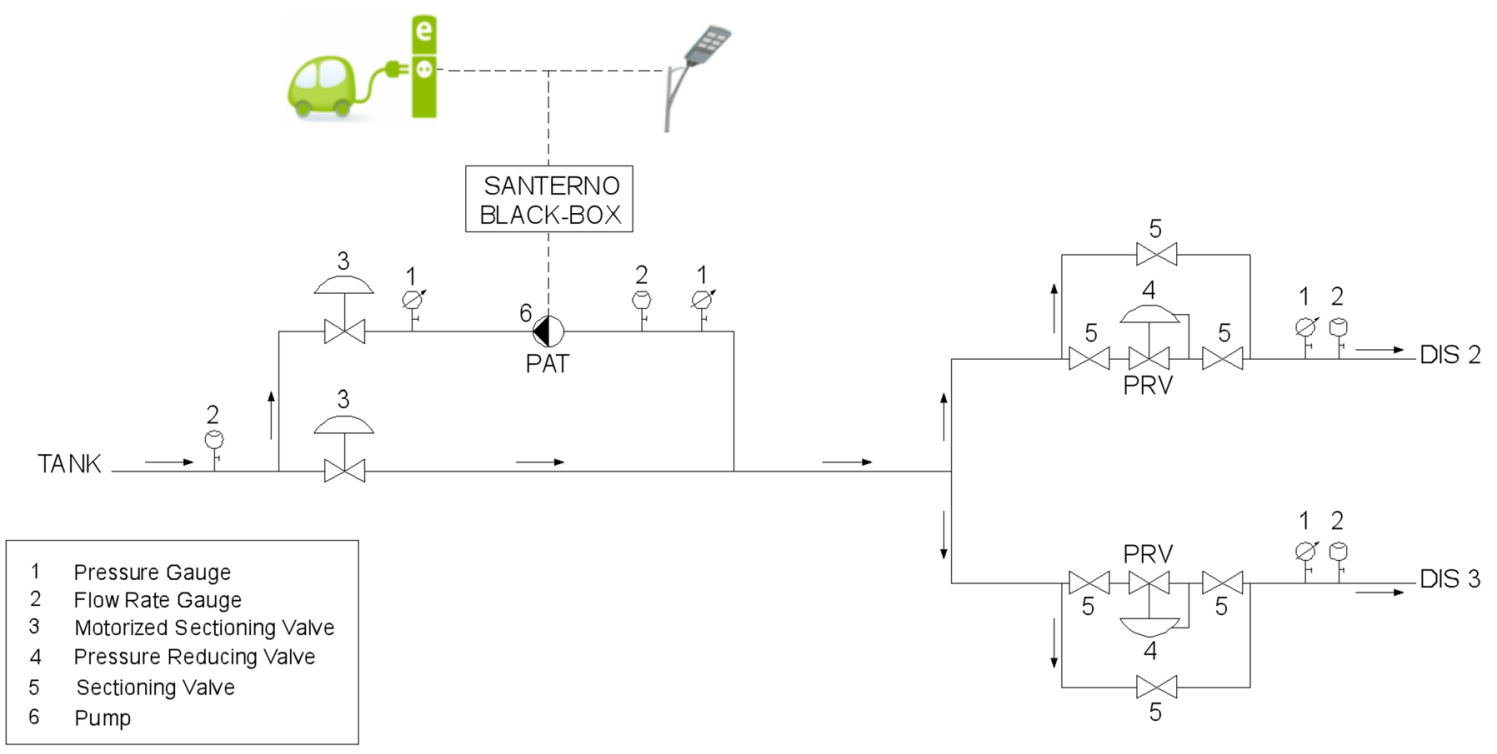

Fig. 7 Sketch of the PAT installation system

by-pass pipe; likewise, two pressure gauges control pressure upstream and downstream of the by-pass.

Downstream the PAT, the existing PRVs are still in line in the system, both equipped with sectioning valves to permit to by-pass the same or not. While the valve of the DIS3 will never go into operation, except for conditions of malfunction of the PAT, the one on the DIS2 will need to be reprogrammed according to the new pressure range in which to operate, to contain excessive downstream water pressure as above explained. Energy produced by PAT and later addressed into the "Black-box Generator" is then used to supply the recharging point and, in case of excessive energy production or absence of electric request by EVs, to supply a street light near the recharging point. The system supplies in priority the EVs recharging point, if necessary the streetlamp equipped with a daylight detector, and energy in excess will be channeled into the power grid. Moreover, in case of need, the EVs' recharging point will also be supplied from the grid.

All the equipment connected to by-pass line construction and mechanical work related to the PAT system will be introduced in a new valve chamber with a sufficient space to contain the equipment.

Starting from the main pipe position and considering the purpose of the system plant, the location for the valve 
chamber and the recharging point will be in a parking zone into the urban area (Figs. 8, 9).

Directive 2014/94/EU suggested Union-wide common connectors for electric vehicles (Type 2 and Combo 2) with the aim to allow multistandard recharging. According to the technical specifications dictated by the above-mentioned directive, the adopted recharging point for this study was a single-phase column adjustable to $3.7 \mathrm{~kW}$, with 2 type 2 sockets and RFID reader.

The management of the users, enabled to use the charging stations, is obtained thanks to the RFID card. Moreover, the recharging point is equipped with a monitoring software, useful for accounting all the recharges performed by different electric vehicles.

\section{Economic analysis}

PAT is a useful and low-cost solution to produce energy from a WDN. Nevertheless, it is necessary to conduct a detailed and careful economic evaluation to compare the expected costs and benefits by means of economic analysis criteria.

The economic analysis was achieved comparing costs and revenues for the expected life of the PAT. The costs were subdivided into capital costs that sum all the equipment costs necessary to realize the system; the annual operation cost, due to the maintenance of every devices and civil works, was estimated, respectively, equal to $0.5 \%$ and to $2.5 \%$ of

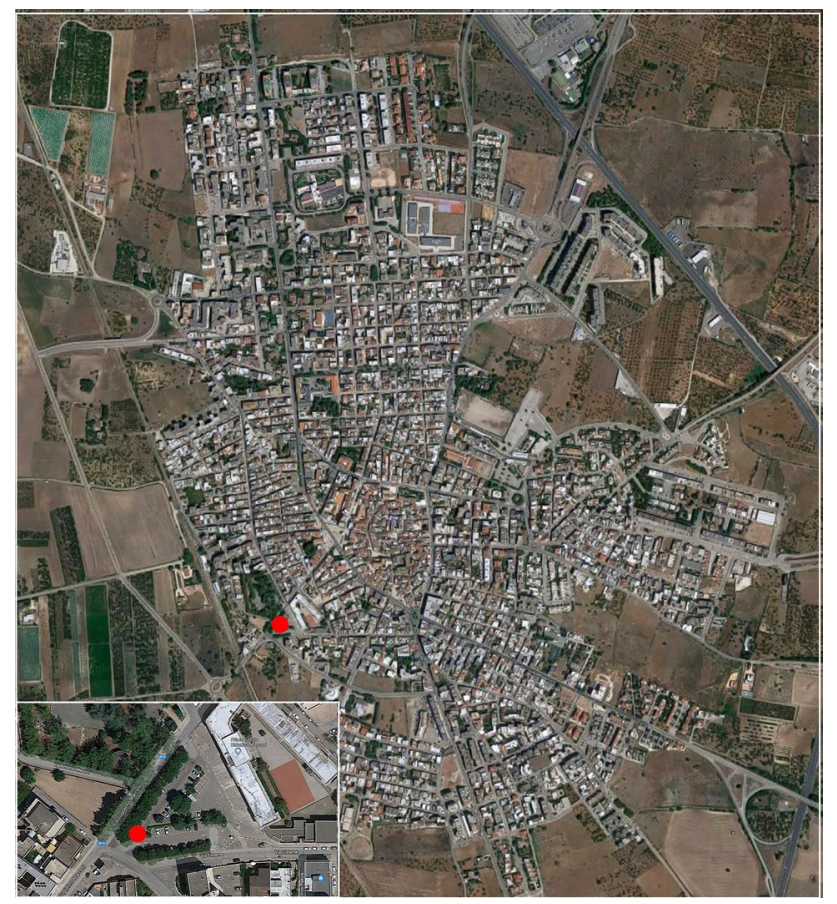

Fig. 8 Installation point for the recharging point

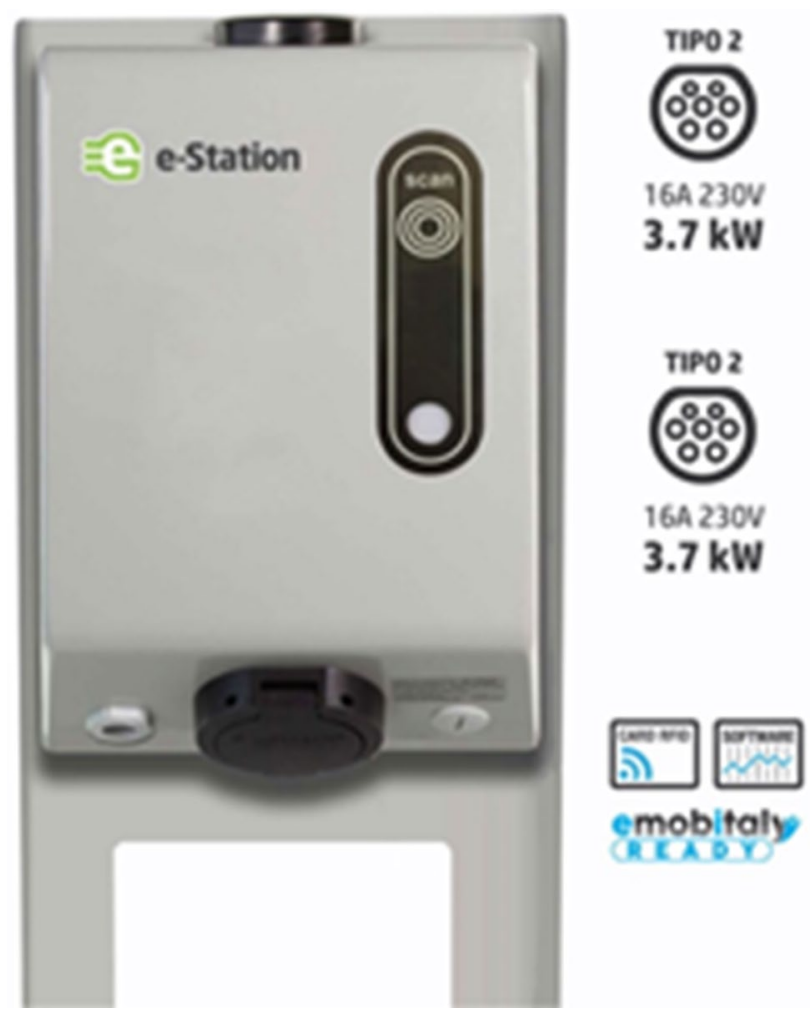

Fig. 9 Recharging point

the capital costs; finally, the repositioning costs related to the substitution of the equipment were considered. Other costs consisting in research and in the development of a first prototype, set-up, etc., were not accounted in this work.

Table 2 summarizes capital costs considered into the present study: hydraulic equipment includes the PAT, and all the necessary hydraulic devices already described by Fig. 7, civil works essential to build a new valve chamber to contain the equipment and again the electric devices that are, respectively, the Santerno Black-box composed by two electric frequency converters and a PLC-based control system, the EVs' recharging point and the street lamp.

Table 2 Implementation costs of the PAT configuration

\begin{tabular}{ll}
\hline Items & Cost $(€)$ \\
\hline Pat cost & $6926 €$ \\
Hydraulic system (by-pass, valves, pipes, etc.) & $24,526 €$ \\
Civil works & $15,000 €$ \\
Santerno Black-box & $5878 €$ \\
Recharging point for EVs (Combo 2) and light point & $4000 €$ \\
Electronic device and SCADA connection device & $14,030 €$ \\
Total cost & $70,359 €$ \\
\hline
\end{tabular}


The useful life of the project has been estimated in 15 years, equal to the duration of the mechanical/electrical equipment life-cycle.

A cost-benefit analysis was conducted using both the statistical method, evaluating the definition of payback period and return of investment (ROI), and the dynamic approach, thanks to net present value (NPV) and Internal rate of return (IRR). The latter, unlike the former, take into account the total costs and benefits for the entire useful life of the equipment and the moment when the cash inflows occur. A comprehensive cost-benefit analysis must also include the estimation of the environmental benefits and their revenues derived from the use of the PAT, able to dissipate excess water pressure of a WDN and to convert it into electricity, in order to supply a recharging point for EVs. Annual energy production by the PAT system was estimated at $57,587 \mathrm{kWh} /$ year and the annual revenue for this produced energy was computed as 13,245 €/year. Moreover, considering the hypothesized useful life, total energy production was at $836,806 \mathrm{kWh}$ and the relative revue is $198,675 €$. Furthermore, considering the recovery of water losses due to the pressure reduction into the WDN and thanks to the information of the water management company, able to compare the volumes introduced into the network and those invoiced before and after the installation of PRVs, a volume of water savings of about $0.5 \mathrm{Mm}^{3} /$ year was estimated. Considering a water tariff of $0.20 € / \mathrm{m}^{3}$, a full-recovery cost of water of $100,000 € /$ year was obtained.

The evaluation of reduction in carbon dioxide $\left(\mathrm{CO}_{2}\right)$ emissions due to green energy production was estimated at $345,522 \mathrm{~g} \mathrm{CO}_{2}$, considering an emission factor of $450 \mathrm{~g} /$ $\mathrm{kWh}$ obtained by Italian national data for the last 10 years [34].

Table 3 Economic analysis results

\begin{tabular}{ll}
\hline Economic indicators & Value \\
\hline Payback Period & 0.62 years \\
ROI & $158 \%$ \\
B/C & 15.61 \\
NPV & $979,281 €$ \\
IRR & $60.57 \%$ \\
\hline
\end{tabular}

Results are summarized in Tables 3 and 4. Based on the capital costs and on the total amount revenues for each day of operation, the payback period was estimated of about 7.5 months, a very short time compared with classical turbine and overall very short compared to useful life of the investment. Return of investment (ROI) evaluates the amount of return on an investment respect to the investment's cost. To calculate ROI, the benefit (or return) of an investment was divided by the cost of the same investment and the result was expressed as a percentage, in this case the ROI amounts to $158 \%$. The benefit/cost ratio (B/C) compares the actual values of benefit and costs; in particular, a value of 15.61 highlights the degree of desirability of this equipment. NPV, defined as the sum that results when the expected investment and operating costs of the project are deducted from the discounted value of the expected revenues, amounts to $979,281 €$. IRR conveys the discount rate that makes the NPV equal to zero and for this study amounts to $60.57 \%$. The last one is an essential economic parameter, since measures the capacity of the net revenues to remunerate the investment cost.

However, the cost-benefit analysis reported here is useful to provide only a preliminary estimation of the cost-effectiveness of the proposed intervention. Strictly speaking, other benefits such as $\mathrm{CO}_{2}$ reduction [35] and other external costs should also be included in the same analysis.

In an economic analysis, it is necessary to compare a project with other possible alternatives. In this particular case, only an option can be compared and it consists with PRVs' application in the WDN, since the starting point is to guarantee water pressure control and pipe leakages reduction. The total cost faced by the water management for the existing PRVs has been estimated to amount to $45,000 €$ that, compared with that of PAT system (Table 2), shows how a PAT system can be easily adopted like a valid alternative. A similar choice adopted by water managers, when allowed by the hydraulic conditions, is a new way to generate and to use in the same place green energy for the EVs recharge. Moreover, a similar installation plant, similar to PRVs, leads to a reduction in the rate of breaking of valves and pipes. Even this last aspect has not been included in the economic evaluation and could improve results as well as the increased satisfaction degree of customers due to an improvement of the network pressure pattern.
Table 4 Environmental benefits and revenues of the PAT system

\begin{tabular}{llc}
\hline Item & $\begin{array}{l}\text { Environmental } \\
\text { benefit }\end{array}$ & Revenue $(€)$ \\
\hline Annual energy production by the PAT system $(\mathrm{kWh} /$ year $)$ & 57,587 & 13,245 \\
Energy production by the PAT system $(\mathrm{kWh})$ & 836.806 & 198,675 \\
Reduction of CO2 emissions from energy saving $\left(\mathrm{g} \mathrm{CO}_{2}\right)$ & 345,522 & \\
Water saving due to pressure reduction by the PAT system $\left(\mathrm{m}^{3} /\right.$ year) & 500,000 & 100,000 \\
\hline
\end{tabular}




\section{Conclusions}

The attention to the environment and to the pollution reduction, including atmospheric emissions, is driving the entire world community towards the research of new renewable energy sources. At the same time, the forthcoming future sees an ever-increasing number of EVs, and consequently, this increase will lead to a greater demand for electricity.

In light of the above, the focus of this study was to reduce the energy demand on the grid, due to EVs by locally producing the charging power in a 'green' manner through the available excess pressure in a WDN. Energy provided by the available excess water pressure can be recovered using PATs. As an alternative, pressure reduction strategies and energy dissipation (PRVs) are applied with the aim to control and to reduce pipe leaks.

With this purpose, the functioning of a real WDN in Casamassima (Italy) was analyzed, starting from the existing presence of two PRVs. The configuration designed considers replacing the existing PRVs with a PAT, to recover all the possible energy, converting the hydraulic head obtained as the difference between the upstream pressure head and the required backpressure. However, the PAT functioning scheme guarantees minimum backpressure already defined by AQP and implemented into the regulation patterns of the existing PRVs.

The power station consists of a PAT, a "Black-box Generator", composed by electric frequency converters, and a PLC-based control system able to control the flow rate regulation valve and the generator itself. The generator, managed by the control system, gives the energy produced by the PAT to the electrical grid with values of voltage and frequency according to the grid standards. This peculiar "generator" system allows to give energy to the electrical grid with any rotational speed of the PAT. Energy produced is then used to supply the recharging point for EVs and/or to supply a street light near the recharging point.

All numerical evaluations regarding the pump performance in a turbine mode have been based on several bibliographic studies, that investigate pumps very similar to that identified in this study. The economic analysis confirms the goodness of such installation plant, reinforced by a very short payback period. Moreover, a comparison between the total cost of the existing PRVs system and that prosed in this study leads to believe that such installations can be easily considered an alternative to PRVs.

Installation systems similar to those proposed may be an encouragement to induce local administrations to sign agreements with the water management authority, to implement incentive policies resulting in the generation of a "green and free" charging infrastructure at zero energy cost.

\section{Compliance with ethical standards}

Conflict of interest On behalf of all authors, the corresponding author states that there is no conflict of interest.

Open Access This article is distributed under the terms of the Creative Commons Attribution 4.0 International License (http://creativecommons.org/licenses/by/4.0/), which permits unrestricted use, distribution, and reproduction in any medium, provided you give appropriate credit to the original author(s) and the source, provide a link to the Creative Commons license, and indicate if changes were made.

\section{References}

1. Bartlett, A., Marcicki, J., Onori, S., Rizzoni, G., Yang, X.G., Miller, T.: Electrochemical model-based state of charge and capacity estimation for a composite electrode lithium-ion battery. IEEE Trans Control Syst Technol 24(2), 384-399 (2016)

2. Papavasiliou, A., Oren, S.S.; Sidhu, I.; Kaminsky, P.: Renewable energy supply for electric vehicle operations in California. IAEE 32nd International Conference Monday, June 22nd, 2009 San Francisco CA, USA; June 21-24 (2009)

3. Liu, L., Kong, F., Liu, X., Peng, Y., Wang, Q.: A review on electric vehicles interacting with renewable energy in smart grid. Renew Sustain Energy Rev 51, 648-661 (2015)

4. Šare, A., Krajačić, G., Pukšec, T., Duić, N.: The integration of renewable energy sources and electric vehicles into the power system of the Dubrovnik region. Energy Sustain Soc 5, 27 (2015)

5. Carravetta, A., Del Giudice, G., Fecarotta, O., Ramos, H.M.: Energy production in water distribution networks: a PAT design strategy. Water Resour Manag 26, 3947-3959 (2012)

6. Carravetta, A., Giugni, M.: Functionality factors in the management and rehabilitation of water networks. In: Fondazione, A.M.G.A., Bertola, P., Franchini, M. (eds.) Management of water networks, Proc Conf Efficient Management of Water Networks. Design and Rehabilitation Techniques, pp.77-94, Ferrara, Italy, May 2006, pp. 77-94. Franco Angeli Ed., Milano (2009)

7. Karadirek, I., Kara, S., Yilmaz, G., Muhammetoglu, A., Muhammetoglu, H.: Implementation of Hydraulic Modelling for WaterLoss Reduction Through Pressure Management. Water Resour Manage 26(9), 2555-2568 (2012)

8. Carravetta, A., Derakhshan Houreh, S., Ramos, H.M.: Pumps as Turbines. Springer, Berlin (2018)

9. Samora, I.; Manso, P.; Franca, M.J.; Schleiss, A.J.; Ramos, H.M.: Feasibility assessment of micro-hydropower for energy recovery in the water supply network of the city of Fribourg. Sustainable Hydraulics in the Era of Global Change Liege, Belgium, 27-29 July 2016, Erpicum et al. (Eds.), Taylor \& Francis Group, London (2016)

10. Muhammetoglu, A., Nursen, C., Karadirek, E., Muhammetoglu, H.: Evaluation of performance and environmental benefits of a full-scale pump as turbine system in Antalya water distribution network. Water Sci Technol Water Supply 18, 130-141 (2017)

11. Rossi, M., Righetti, M., Renzi, M.: Pump-as-Turbine for energy recovery applications: the case study of an aqueduct. Energy Procedia 101, 1207-1214 (2016)

12. Jain, S.V., Patel, R.N.: Investigations on pump running in turbine mode: a review of the state-of-the-art. Renew Sustain Energy Rev 30, 841-868 (2014)

13. Lueneburg, R.; Nelson, R.M.: Hydraulic Power Recovery Turbines. In Lobanoff V.S. et al. (eds.) Pumps: Design and Applications, pp. 246-282 Ross (Eds.), Texas, USA (1985) 
14. Fernandez, J., Blanco, E., Parrondo, J., Stickland, M.T., Scanlon, T.J.: Performance of a centrifugal pump running in inverse mode. Proc Inst Mech Eng Part A J Power Energy 218(4), 265-271 (2004)

15. Nautiyal, H., Varun, V., Kumar, A., Yadav, S.: Experimental investigation of centrifugal pump working as turbine for small hydropower systems. Energy Sci Tech 1(1), 79-86 (2011)

16. Dannier, A.; Del Pizzo, A.; Giugni, M.; Fontana, N.; Marini, G.; Proto, D.: Efficiency evaluation of a micro-generation system for energy recovery in water distribution networks. Proceedings of the 5th International Conference on Clean Electrical Power. Renewable Energy Resources Impact (ICCEP15), Taormina, Italy, pp. 689-694 (2015)

17. Jain, S.V., Swarnkar, A., Motwani, K.H., Patel, R.N.: Effects of impeller diameter and rotational speed on performance of pump running in turbine mode. Energy Convers Manag 89, 808-824 (2015)

18. Pugliese, M., De Paola, F., Fontana, N., Giugni, M., Marini, G.: Experimental characterization of two Pumps as Turbines for hydropower generation. Renew Energy 99, 180-187 (2016)

19. Carravetta, A., Golia, U.M., Fecarotta, O.: Energy Saving in a Water Supply Network by Coupling a Pump and a Pump as Turbine (PAT) in a Turbopump. Water 9, 62 (2017)

20. Buono, D., Frosina, E., Mazzone, A., Cesaro, U., Senatore, A.: Study of a pump as turbine for a hydraulic urban network using a tridimensional CFD modeling methodology. Energy Procedia 82, 201-208 (2015)

21. Ramos, H.M., Borga, A., Simão, M.: New design solutions for low-power energy production in water pipe systems. Water Sci Eng 2(4), 69-84 (2009)

22. Barrio, F.R., Blanco, E., Parrondo, J., Marcos, A.: Numerical investigation of a centrifugal pump running in reverse mode. Proc Inst Mech Eng Part A J Power Energy 224(3), 373-381 (2010)

23. Williams, A.A.: The turbine performance of centrifugal pumps: a comparison of prediction methods. J Power Energy 208, 59-66 (1994)

24. Sinagra, M., Sammartano, V., Morreale, G.: A new device for pressure control and energy recovery in water distribution networks and tullio tucciarelli. Water 9(309), 2-14 (2017)
25. Jain, S.V., Patel, R.N.: Investigations on pump running in turbine mode: a review of the state-of-the-art. Renew Sustain Energy Rev 30, 841-868 (2014)

26. Chapallaz, J.M., Eichenberger, P., Fischer, G.: Manual on pumps used as turbines. MHPG Series, Germany, Volume 11 (1992)

27. Balacco, G., Carbonara, A., Gioia, A., Iacobellis, V., Piccinni, A.F.: Evaluation of Peak Water Demand Factors in Puglia (Southern Italy). Water 9, 96 (2017)

28. Carravetta, A., Conte, M.C., Fecarotta, O., Ramos, H.M.: Evaluation of PAT Performances by Modified Affinity Law. Procedia Eng 89, 581-587 (2014)

29. Yang, S.S., Derakhshan, S., Kong, F.Y.: Theoretical, numerical and experimental prediction of pump as turbine performance. Renew Energy 48, 507-513 (2012)

30. Stepanoff, A.J.: Centrifugal and axial flow pumps: theory, design and application. Wiley, New York (1948)

31. Sharma, K.: Small hydroelectric project-use of centrifugal pumps as turbines, technical report. Kirloskan Electric Co., Bangalore (1985)

32. Derakhshan, S., Nourbakhsh, A.: Experimental study of characteristic curves of centrifugal pumps working as turbines in different specific speeds. Exp Therm Fluid Sci 32(3), 800-807 (2008)

33. Derakhshan, S., Nourbakhsh, A.: Theoretical, numerical and experimental investigation of centrifugal pumps in reverse operation. Exp Therm Fluid Sci 32(8), 1620-1627 (2008)

34. ISPRA: Fattori di emissione atmosferica di $\mathrm{CO} 2$ e sviluppo delle fonti rinnovabili nel settore elettrico, Gennaio 2015, 212 (2015) (in Italian)

35. Moore, F.C., Diaz, D.B.: Temperature impacts on economic growth warrant stringent mitigation policy. Nat Clim Chang $\mathbf{5}$, 127-131 (2015)

Publisher's Note Springer Nature remains neutral with regard to jurisdictional claims in published maps and institutional affiliations. 